\title{
Outcome of Calcaneoplasty in Insertional Achilles Tendinopathy
}

\author{
Lim SM, MBBS, Yeap EJ*, MS Ortho, Lim YW*, B Pharm, M Yazid, MMed Ortho \\ Department of Orthopaedics and Traumatology, Hospital Tuanku Fauziah, Kangar, Perlis \\ *Perlis Clinical Research Centre, Hospital Tuanku Fauziah, Kangar, Perlis
}

\begin{abstract}
Background: Insertional Achilles tendinopathy may be associated with Haglund's deformity and result in chronic pain. It is usually refractory to conservative management. The aim of this study was to assess the outcome of calcaneoplasty performed in our hospital. Methods: Eight patients were prospectively evaluated pre- and postoperatively using the American Orthopaedic Foot and Ankle Society (AOFAS) Hindfoot Score, Visual Analogue Scale (VAS) and Short Form (SF) 36, as well as satisfaction rate. Average follow-up duration was 12.4 months. Results: The mean pre and post-operative AOFAS scores were significantly improved at three and six months. SF 36 improved in most parameters. The postoperative VAS score improved significantly and was 0 at 2 years. The procedure was rated as good to excellent by seven of the subjects. Conclusion: We recommend calcaneoplasty for the treatment of insertional Achilles tendinopathy.
\end{abstract}

Key Words:

Calcaneoplasty, Insertional Achilles tendinopathy,

Haglund's deformity

\section{INTRODUCTION}

Insertional Achilles tendinopathy refers to pathology at the insertion of the Achilles tendon onto the calcaneum ${ }^{1}$. It occurs as a result of recurrent stress placed upon the attachment site of the Achilles tendon, leading to inflammation, micro tears, swelling and pain. Overuse and poor training habits are important etiologic factors, considering the prevalence of this condition among runners ${ }^{2,3}$. Whilst mechanical loading has been deemed to be a major risk factor, factors such as advancing age, dyslipidaemia, being of the male sex and high body mass index have been implicated as well ${ }^{4}$. In addition, varus hindfoot malalignment is also associated with Achilles tendon disorders ${ }^{5}$. The diagnosis of insertional Achilles tendinopathy is often clinical, based on a triad of symptoms: pain at the site of insertion of Achilles tendon, accompanied by swelling (which may be due to retrocalcaneal bursitis) and impaired performance of the diseased tendon ${ }^{6}$. In addition, there may be radiological findings such as Haglund's deformity which is an enlargement of the posterosuperior prominence of the calcaneum and/or calcaneal spurs ${ }^{7}$.

This condition is often initially treated with rest, the use of heel pads or open-back shoes, analgesics such as nonsteroidal anti-inflammatory drugs (NSAIDs) and physiotherapy ${ }^{8}$. All are aimed at eliminating pressure and relieving inflammation of the symptomatic area. Eccentric exercises have some proven benefits for the treatment of chronic insertional Achilles tendinopathy, provided it is taught and supervised by trained personnel. These exercises are designed to provide a strong, controlled, mechanical force to the Achilles tendon ${ }^{9}$.

When conservative methods fail, surgery may be performed to repair and reconstruct the degenerative Achilles tendon. Although some centres use an endoscopic method, most opt for an open approach, with either a longitudinal or transverse incision ${ }^{10}$. The diseased tendon which may be partially or totally detached from its insertion, is debrided and ossified tissue and bony spurs are excised. The tendon is reattached with suture anchors if it is totally detached.

This study was conducted to assess the outcomes of calcaneoplasty performed by a single surgeon at a major hospital. The outcome was evaluated on the American Orthopaedic Foot and Ankle Society (AOFAS) hind foot scoring system and the Short Form (SF 36) Health Survey.

\section{MATERIALS AND METHODS}

Ten patients were prospectively recruited from June 2009 to October 2011. Inclusion criteria were: diagnosis of insertional Achilles tendinopathy; consent for surgical intervention for their chronic pain; fitness for surgery. Of the ten patients, seven were female, and all were of varying levels of fitness. While conservative measures were attempted and failed in most of these patients, some had been advised to undergo surgery at initial presentation due to unbearable pain and severe impairment of function. Two 
patients were excluded because although they had initially consented for surgery, they refused at the very last minute for personal and social reasons. The remaining eight had a mean age of 57 years and a mean BMI of 29 (Table I).

The procedure includes making a $4 \mathrm{~cm}$ longitudinal incision at the heel (either central or paracentral) followed by total detachment of the Achilles tendon, debridement then reattachment using suture anchors. In addition, the inflamed retrocalcaneal bursa is usually removed with a rongeur, and the bony prominence at the posterosuperior aspect of the calcaneum is osteotomised prior to reattachment of the Achilles tendon (Figure 1). The procedure was done with or without endoscopic gastrocnemius release depending on whether there was gastrocnemius equinus contracture ${ }^{11}$.

Following surgery, the patient was either put on a pneumatic air cast or below knee Plaster of Paris (POP) cast and instructed not to bear weight for at least 6 weeks. Partial to full weight bearing was then progressively attained.

Outcomes were assessed with AOFAS scoring and the SF 36 Health Survey, administered once preoperatively; then at 3 months, 6 months and 2 years postoperatively. Data was analysed using the Wilcoxon signed rank test. Data for analysis was extracted from outpatient records and from personal interviews.

\section{RESULTS}

The duration of surgery ranged from 45 minutes to two hours. The average hospital stay after the procedure was three days. Preoperatively, AOFAS scores ranged from 31 to 87 , with the mean being 58 . At 3 months postoperatively, the mean score improved to 83.6 (range, 55-98). At 6 months, the mean AOFAS score was 81.3 (range, 68-100). Only three patients have currently completed the recommended 2-year follow up duration, and it is interesting to note that all three have a score of 100 (Table II). Wilcoxon signed rank analysis of the AOFAS scores were significant ( $p$ values $<0.017$ at three months postoperative visit and $\mathrm{p}<0.028$ for six months postoperative visit).

The range for the preoperative VAS pain score was 2 to 8 . The mean pain score improved from a mean preoperatively of 5.9 to 1.9 at the 3 month postoperative visit (median improved from 6 to 1). With the exception of one outlier, the 7 participants had a pain score ranging from 0 to two at the three month follow-up visit. The mean VAS at six months was 2.3, and at two years was 0 (Table III). Using the Wilcoxon signed rank test to compare preoperative with 3 and 6 month follow-up VAS, there was a statistically significant ( $p$ value $<0.021$ and $p<0.027$ respectively) difference.

On the SF 36 Health Survey, 8 parameters considered are scored on a scale of 0 to 100 , with 100 being the maximal level of possible function. Analysing the preoperative and three month postoperative scores, a positive trend was identified for most factors, with a mean $17 \%$ increase for overall physical function ( $48 \%$ to $65 \%$ ), $19 \%$ reduction in role limitation due to physical health (34\% to $53 \%), 16 \%$ increase in energy (63\% to $79 \%), 2 \%$ increase each in emotional well-being (84\% to $86 \%$ ) and social functioning ( $75 \%$ to $77 \%$ ), $16 \%$ in pain reduction $(57 \%$ to $73 \%$ ), and $22 \%$ in general health $(53 \%$ to $75 \%)$. However, there was a $17 \%$ increase in role limitations due to emotional problems (92\% to $75 \%$ ). (Figure 2 and 3 )

The duration of follow-up ranged from a minimum of three months to a maximum of two years. Average follow-up duration was 12.4 months. At three months, two patients felt that the operation totally met their expectation, for another five patients it almost totally did, while the remaining one claimed that it did not quite meet her expectation (Table IV). For the same duration, three patients rated the overall results of the surgery as "excellent", 2 as "very good", and another two as "good" while one patient felt it was "poor". The three patients who completed a 2 year follow up reported that the surgery was "very good" to "excellent", and that it had either totally or almost totally met their expectations (Table V).

One complication was wound breakdown and chronic osteomyelitis of the calcaneum. This patient was later diagnosed to have a stitch granuloma. Another patient had a painful scar.

\section{DISCUSSION}

Several studies have been published on the subject of calcaneoplasty. Most were not conducted in an Asian setting, and therefore could not really be generalised to our local population. Research customised to local settings is important in view of the cultural and social practice among Asians, which is associated with a higher tolerance of pain, thereby presenting for surgery at later stages of deformity or disease. In early stages, complete detachment of the Achilles tendon may not be a necessary part of treatment. At its initial presentation, surgery is usually not even required, as a systemic review by Kearney et al. showed that conservative methods should be used before resorting to surgical intervention $^{12}$. In addition, we may have a higher prevalence of tight gastrocnemius muscles, chiefly due to our daily habits, where kneeling and sitting cross-legged for prolonged periods, such as during meditation, is common practice.

Insertional Achilles tendinopathy may be quite effectively treated with conservative measures as described earlier, when presented at early stages. However, most patients usually present much later such that irreparable damage has already been done. This could be due to under diagnosis of this condition, as well as patients' tendency to pursue traditional treatment in our local setting. 
Table I: Demographic characteristics of patients participating in study

\begin{tabular}{|llcc|}
\hline & & Male & Female \\
\hline Age Group & $45-49$ & & $1(12.5 \%)$ \\
& $50-54$ & & $3(37.5 \%)$ \\
& $55-59$ & $1(12.5 \%)$ & $1(12.5 \%)$ \\
Race & $60-64$ & $1(12.5 \%)$ & $5(62.5 \%)$ \\
& $65-69$ & $1(12.5 \%)$ & $4(50 \%)$ \\
Occupation & Malay & $2(25 \%)$ & $1(12.5 \%)$ \\
& Indian & $1(12.5 \%)$ & $2(25 \%)$ \\
Frequency of Exercise & Homemaker & & $3(37.5 \%)$ \\
& Retiree & $3(37.5 \%)$ & \\
Rody Mass Index & Occasionally & & $2(25 \%)$ \\
& Regular & $1(12.5 \%)$ & $2(25 \%)$ \\
& 18.5 -25 (Normal) & $1(12.5 \%)$ & $2(25 \%)$ \\
\end{tabular}

Table II: Results of American Orthopaedic Foot and Ankle Society (AOFAS) Hind Foot Scoring

\begin{tabular}{|lcccc|}
\hline & Pre Op & 3 Months & 6months & 2 Years \\
\hline LK & 36 & 79 & NA & 100 \\
FMN & 65 & 90 & 88 & 100 \\
SMF & 52 & 87 & 79 & 100 \\
KMN & 72 & 85 & 75 & NA \\
NA & 31 & 98 & 78 & NA \\
SM & 57 & 55 & 68 & NA \\
OP & 87 & 97 & 100 & NA \\
NJ & 65 & 78 & NA & 100 \\
MEAN & 58.1 & 83.6 & 81.3 & 0.109 \\
\hline
\end{tabular}

Table III: Results of Visual Analogue Scale (VAS)

\begin{tabular}{|lcccc|}
\hline & Pre OP & 3months & 6months & 2 Years \\
\hline LK & 7 & 2 & NA & 0 \\
FMN & 8 & 1 & 1 & 0 \\
SMF & 5 & 0 & 4 & 0 \\
KMN & 5 & 1 & 2 & NA \\
NA & 8 & 0 & 2 & NA \\
SM & 7 & 9 & 5 & NA \\
OP & 2 & 0 & 0 & NA \\
NJ & 5 & 2 & NA & 0 \\
MEAN & 5.9 & 1.9 & 2.3 & 0.109 \\
$P$ value & - & 0.021 & 0.027 & \\
\hline
\end{tabular}

Table IV: Expectation met for surgery

\begin{tabular}{|lccc|}
\hline & $\mathbf{3}$ months & $\mathbf{6}$ months & $\mathbf{2}$ years \\
\hline Yes, totally & $2(25 \%)$ & $3(50 \%)$ & $1(33.3 \%)$ \\
Yes, almost totally & $5(62.5 \%)$ & $1(16.7 \%)$ & $2(66.7 \%)$ \\
Yes, quite a bit & 0 & $1(16.7 \%)$ & 0 \\
More or less & 0 & 0 & 0 \\
No, not quite & $1(12.5 \%)$ & $1(16.7 \%)$ & 0 \\
No, far from it & 0 & 0 & 0 \\
No, not at all & 0 & 0 & 0 \\
\hline
\end{tabular}


Table V: Satisfaction with surgery

\begin{tabular}{|lccc|}
\hline & $\mathbf{3}$ months & $\mathbf{6}$ months & $\mathbf{2}$ years \\
\hline Excellent & $3(37.5 \%)$ & $1(16.7 \%)$ & $2(66.7 \%)$ \\
Very good & $2(25 \%)$ & $2(33.3 \%)$ & $1(33.3 \%)$ \\
Good & $2(25 \%)$ & $2(33.3 \%)$ & 0 \\
Fair & 0 & $1(16.7 \%)$ & 0 \\
Poor & $1(12.5 \%)$ & 0 & 0 \\
Terrible & 0 & 0 & 0 \\
\hline
\end{tabular}
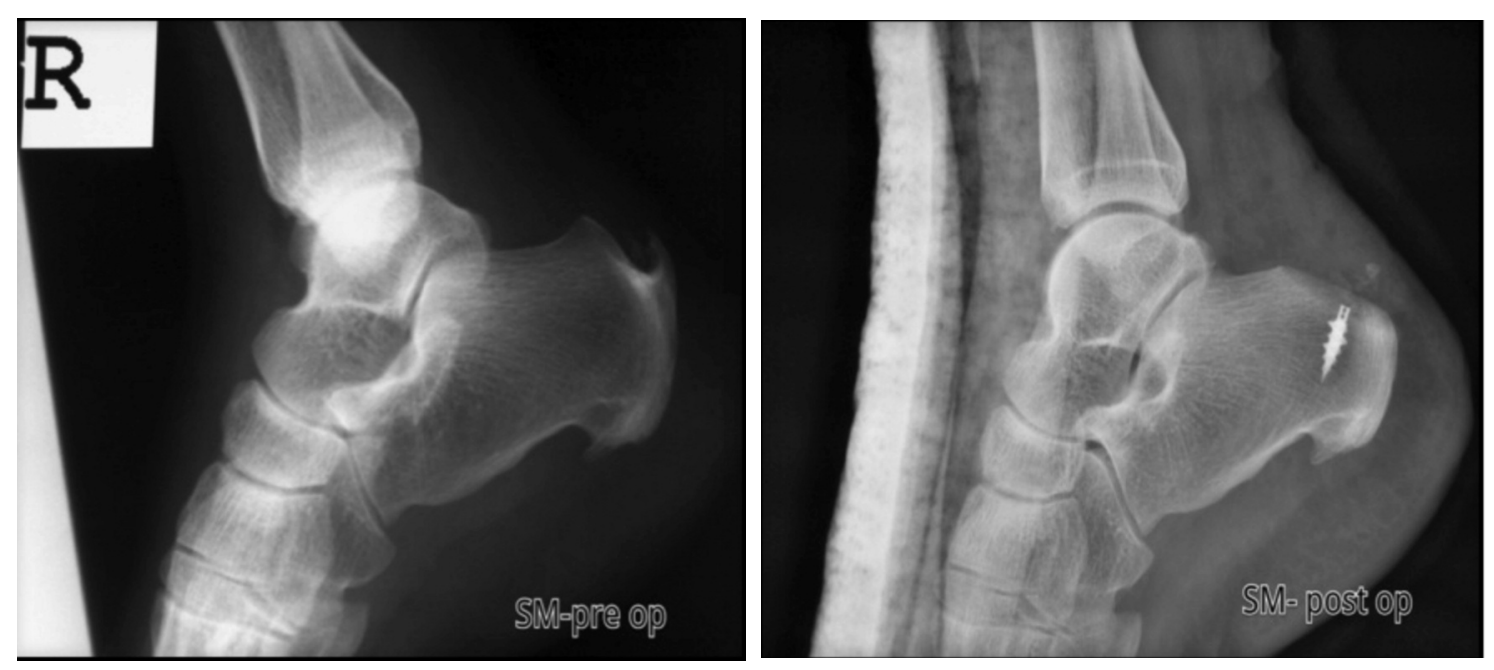

Fig. 1: Radiographs comparing the pre-operative (left image) and post-operative (right image) of a subject, SM, a 48 year-old woman.

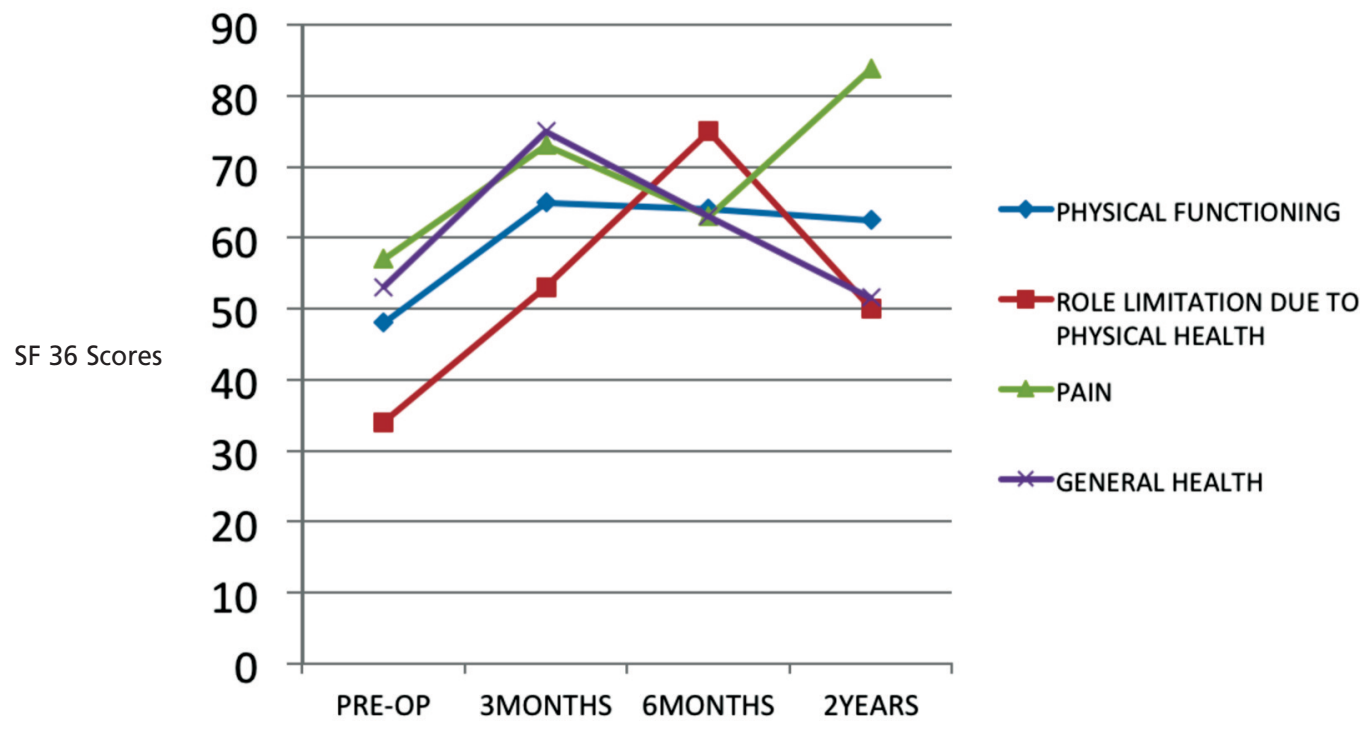

Fig. 2: Graphical depiction of SF 36 scores reflecting physical health. 


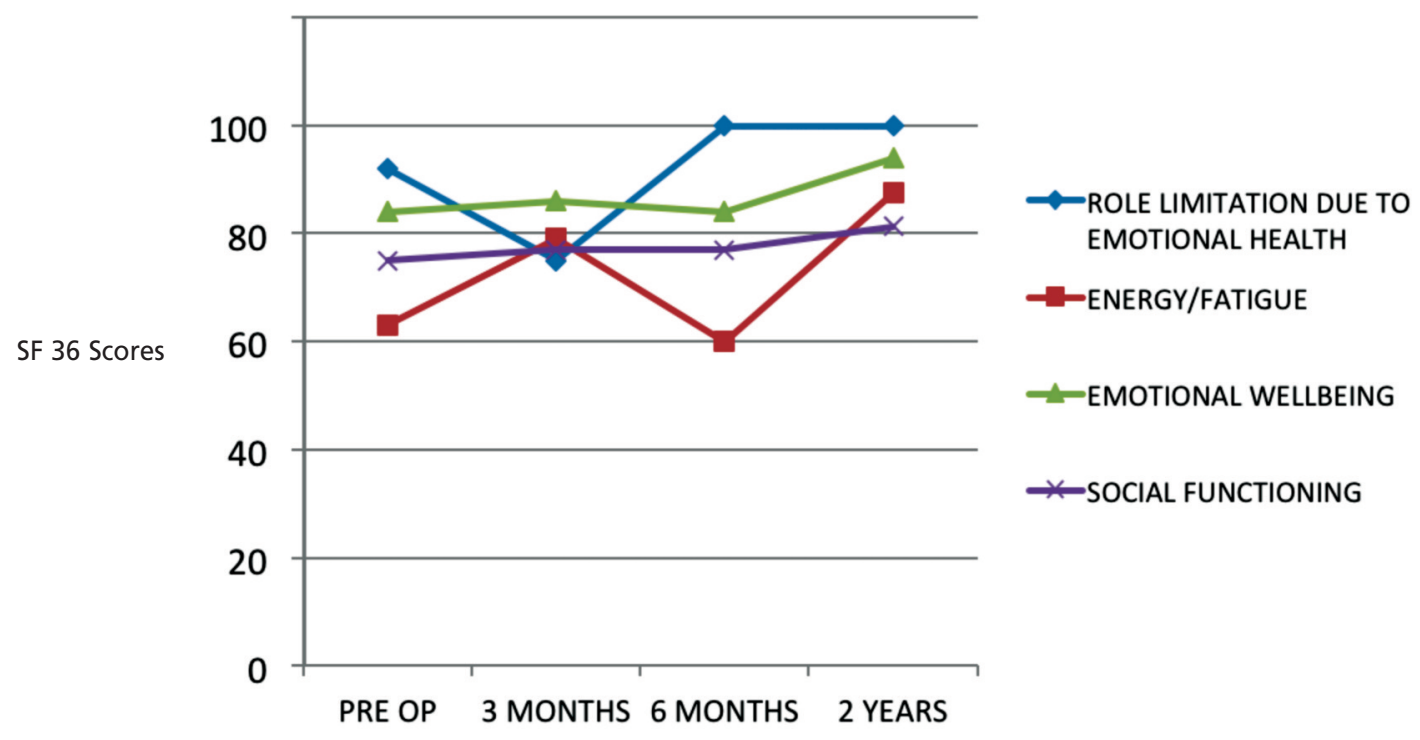

Fig. 3: Graphical depiction of SF 36 scores reflecting emotional health.

Factors assessed include pain utilising the VAS, functional capacities, maximum walking distance, degree of difficulty on different walking surfaces, gait abnormality, range of motion (sagittal motion as well as hind-foot motion), ankle hind-foot stability (anterior posterior and varus-valgus). Our study showed that calcaneoplasty provides prompt pain relief for insertional Achilles tendinopathy, as indicated at the three months follow-up visit where the mean VAS decreased from 5.9 to 1.9 . The difference at two years was insignificant, probably due to the fact that only three subjects completed that stipulated duration of follow up, and yet all 3 had a maximal score of 100 .

The SF 36 Health Survey consists of 36 items, which are aggregated into 8 categories to analyse physical and emotional health, namely: physical functioning; role limitation due to physical health; energy level; emotional well-being; social functioning as a result of pain; general health; role limitations due to emotional problems. Upon analysis of the SF 36 scores we noted that most of the factors showed a positive trend. It is important to take into account the fact that a majority of patients had comorbidities that could affect their SF36 scores and these patients are relatively old, rendering them less able to perform some of the activities evaluated in the survey (i.e., vigorous activities or climbing several flights of stairs). Although the SF 36 Health Survey reflects the general well being of the patient, it is not reflected on the surgical results.

Wagner et al. conducted a similar study, albeit on a much larger scale, with 75 patients ( 81 heels) who were further divided into a detached group (total detachment of the Achilles tendon) and a non-detached group (none or partial detachment of the Achilles tendon) ${ }^{13}$. Factors evaluated included pain, activity limitation, gait change, walking distance, return to sport or work and level of satisfaction. The duration of follow up ranged from 33 months to 47 months. Evaluation took place solely via an interview questionnaire; a standard, universally recognised mode of survey was not employed, and examination was not performed to assess differences in preoperative vs. postoperative range of motion. The authors concluded that for chronic insertional Achilles tendinosis, a reliable and effective treatment is debridement surgery with or without Achilles tendon detachment (followed by reattachment). On the other hand, Nunley et al. conducted a retrospective review of 27 patients who underwent surgery using a central incision technique, and in which isokinetic testing of plantar flexion strength, AOFAS Foot and Ankle Survey and AOFAS Hind Foot Score was assessed; ultimately the authors reported a $96 \%$ satisfaction rate with minimal to no loss of strength ${ }^{14}$.

In the present study, one patient developed wound breakdown and chronic osteomyelitis of the calcaneum that required long-term antibiotics, as well as several debridement procedures, which include the removal of the suture anchors. She extruded out her sutures in the first six weeks. Subsequently, after repeated cultures done were negative, she was diagnosed as having a stitch granuloma via intraoperative findings, in which thick fibrous tissue was found enveloping a strand of non-absorbable suture left in the wound. Stitch or suture granuloma is a rare but recognised complication of surgical procedures, and this is the first incidence that such a complication has been reported following calcaneoplasty. The usual presentation is a mass 
within the surgical site, often mimicking a soft tissue tumour. This entity occurs as a result of the body rejecting the suture material, creating an inflammatory reaction, which eventually resolves once the offending material has been expelled out of the body, whether spontaneously or via surgery. As it is an inflammatory/allergic reaction, rather than an infective one, antibiotic treatment is rarely required. This particular patient, who had no prior history of any form of allergy, was the only one of eight subjects in whom Poly Ether Ether Ketone (PEEK) suture anchors were used to reattach the Achilles tendon. She is currently recovering from her most recent debridement procedure in which more suture remnant was removed, and is otherwise able to ambulate well and is generally satisfied with the calcaneoplasty, sans the mild discomfort felt within the heel. This occasional dull aching sensation, in her opinion, is a small matter compared to the enormous pain relief and functional improvement that followed the initial surgery. Another patient developed a painful scar tissue warranting steroid injection for pain relief and occupational therapy for desensitisation. There was no incidence of sural nerve injury.

Schunck et al. identified several problems commonly faced after open calcaneoplasty, including complications involving the skin, the Achilles tendon, weakness of the calcaneus after removing the posterior-superior bony part, persistent pain, hypertrophy and irritation of the scar, hyperaesthesia in the area of the scar and irritation of the whole heel. Hence, they advocate minimally invasive endoscopic calcaneoplasty as a suitable alternative to the open technique, as it has a lower rate of complications, better visualisation for differentiation of pathology and a short learning curve ${ }^{15}$. This may be possible in the early stages where there is no prominent 'hood ornament', the superior heel spur that occurs as a result of osteophyte formation in response to chronic microtrauma or traction. There are more options available when only a Haglund's deformity with pure insertional Achilles tendinopathy minus the calcification exists. This includes the Kelly-Keck procedure and certainly, endoscopic calcaneoplasty ${ }^{16}$. All cases in the present study had the 'hood ornament' as seen in Figure 1.
Other treatment modalities for insertional Achilles tendinopathy include extracorporeal shockwave therapy, and sonographically guided dextrose injections ${ }^{17-19}$. Research conducted on these methods thus far has been promising, especially for early presentations. Further possible surgical intervention procedures include the use of flexor hallucis longus in the reconstruction of the Achilles tendon or a bonetendon graft harvested from the knee extensor system for Achilles tendon augmentation ${ }^{20-23}$. These procedures are indicated when more extensive debridement is needed, and where whole sections of the Achilles tendon have been removed.

Limitations of this study include the small sample size (making it a non-parametric study), the short duration of follow-up for each patient and the lack of a control group. Moreover, some data could not be included as a few patients defaulted follow-up. This research serves as a pilot project for a prospective study with a larger sample size that can also analyse additional factors such as ability to tip toe or jump postoperatively, and perhaps one to include a comparison group undergoing conservative management.

\section{CONCLUSION}

In conclusion, calcaneoplasty for insertional Achilles tendinopathy showed significant improvement in terms of pain reduction and function as early as three months and sustained until two years following surgery. The procedure contributed to an overall enhancement of the subjects' health condition. The majority of patients in the present study were satisfied with the procedure and felt that it met their expectations. Thus, calcaneoplasty is recommended as the treatment of choice for chronic insertional Achilles tendinopathy that is refractory to conservative measures.

\section{ACKNOWLEDGEMENT}

We wish to thank the Director General of Health, Malaysia for permission to publish this paper. 


\section{REFERENCES}

1. Van Dijk CN, van Sterkenberg MN, Wiegerinck JI, Karlsson J, Mafulli N. Terminology for Achilles tendon related disorders. Knee Surg Sport Tr A 2011; 19(5): 835-41.

2. Krishna Sayana M, Maffulli N. Insertional Achilles tendinopathy. Foot Ankle Clin 2005; 10(2):309-20.

3. Maffulli N, Wong J, Almekinders LC. Types and epidemiology of tendinopathy. Clin Sports Med 2003; 22: 675-92.

4. Gaida JE, Alfredson L, Kiss ZS, et al. Dyslipidemia in Achilles tendinopathy is characteristic of insulin resistance. Med Sci Sports Exerc 2009; 41: 1194-7.

5. Waldecker U, Hofmann G, Drewitz S. Epidemiologic investigation of 1394 feet: coincidence of hind foot malalignment and Achilles tendon disorders. Foot Ankle Surg. 2012; 18(2): 119-23.

6. Witt BL, Hyer CF. Achilles tendon reattachment after surgical treatment of insertional tendinosis using the suture bridge technique: a case series. J Foot Ankle Surg. 2012; 51(4): 487-93.

7. Kang S, Thordarson DB, Charlton TP. Insertional Achilles tendinitis and Haglund's deformity. Foot Ankle Int 2012; 33(6): 48791.

8. Thomas JL, Christensen JC, Kravitz SR, R.W. Mendicino, J.M. Schuberth, J.V. Vanore, et al.The Diagnosis and Treatment of Heel Pain: A Clinical Practice Guideline-Revision 2010. J Foot Ankle Surg 2010; 49: 1-19

9. A Scott, E Huisman, K Khan. Conservative treatment of chronic Achilles tendinopathy. Can Med Assoc J 2011; 183(10): 115965.

10. Maffulli N, Del Buono A, Testa V, Capasso G, Oliva F, Denaro V. Safety and outcome of surgical debridement of insertional Achilles tendinopathy using a transverse (Cincinnati) incision. Bone Joint Surg Br 2011; 93(11): 1503-7.

11. Yeap EJ, Shamsul SA, Chong KW, Sands AK. Simple two-portal technique for endoscopic gastrocnemius recession: clinical tip. Foot Ankle Int 2011; 32(8): 830-3.

12. Kearney R, Costa ML. Insertional achilles tendinopathy management: a systematic review. Foot Ankle Int 2010; 31(8): 689-94.

13. Wagner E, Gould JS, Kneidel M, Fleisig GS, Fowler R, Technique and Results of Achilles Tendon Detachment and Reconstruction for Insertional Achilles Tendinosis. Foot Ankle Int 2006; 27(9): 677-84.

14. Nunley JA, Ruskin G, Horst F Long-term clinical outcomes following the central incision technique for insertional Achilles tendinopathy. Foot Ankle Int 2011; 32(9): 850-5.

15. Schunck J, Jerosch J. Operative treatment of Haglund's syndrome. Basics, indications, procedures, surgical techniques, results and problems. Foot Ankle Surg 2005; 11: 123-30.

16. Maquirriain J Endoscopic Achilles tenodesis: a surgical alternative for chronic insertional tendinopathy. Knee Surg Sports Traumatol Arthrosc 2007 15:940-3.

17. Saxena A, Ramdath S Jr, O'Halloran P, Gerdesmeyer L, Gollwitzer H. Extra-corporeal pulsed-activated therapy ("EPAT" sound wave) for Achilles tendinopathy: a prospective study. J Foot Ankle Surg 2011; 50(3): 315-9.

18. Fridman R, Cain JD, Weil L Jr, Weil L Sr. Extracorporeal shockwave therapy for the treatment of Achilles tendinopathies: a prospective study. J Am Podiatr Med Assoc 2008; 98(6): 466-8.

19. Ryan M, Wong A, Taunton J. Favorable outcomes after sonographically guided intratendinous injection of hyperosmolar dextrose for chronic insertional and midportion achilles tendinosis. Am J Roentgenol 2010; 194(4): 1047-53.

20. Den Hartog BD. Insertional Achilles tendinosis: pathogenesis and treatment. Foot Ankle Clin 2009; 14(4): 639-50.

21. Wong MW, Ng VW. Modified flexor hallucis longus transfer for Achilles insertional rupture in elderly patients. Clin Orthop Relat Res 2005; (431): 201-6.

22. Elias I, Raikin SM, Besser MP, Nazarian LN. Outcomes of chronic insertional Achilles tendinosis using FHL autograft through single incision. Foot Ankle Int 2009; 30(3): 197-204.

23. Philippot R, Wegrzyn J, Grosclaude S, Besse JL. Repair of insertional achilles tendinosis with a bone-quadriceps tendon graft. Foot Ankle Int 2010; 31(9): 802-6. 